\title{
Effectiveness of electrochemotherapy after IFN- $\alpha$ adjuvant therapy of melanoma patients
}

\author{
Andrejc Hribernik ${ }^{1}$ Maja Cemazar², Gregor Sersa², Maša Bosnjak², Marko Snoj¹ \\ ${ }^{1}$ Department of Surgical Oncology, Institute of Oncology Ljubljana, Ljubljana, Slovenia \\ ${ }^{2}$ Department of Experimental Oncology, Institute of Oncology Ljubljana, Ljubljana, Slovenia
}

Radiol Oncol 2016; 50(1): 21-27.

Received 20 October 2015

Accepted 30 November 2015

Correspondence to: Prof. Marko Snoj, M.D., Ph.D., Department of Surgical Oncology, Institute of Oncology Ljubljana, Zaloška 2, SI-1000 Ljubljana, Slovenia. E-mail: msnoj@onko-i.si

Disclosure: No potential conflicts of interest were disclosed.

Background. The combination of electrochemotherapy with immuno-modulatory treatments has already been explored and proven effective. However, the role of interferon alpha (IFN-a) adjuvant therapy of melanoma patients and implication on electrochemotherapy effectiveness has not been explored yet. Therefore, the aim of the study was to retrospectively evaluate the effectiveness and safety of electrochemotherapy after the previous adjuvant treatment with IFN-a in melanoma patients.

Patients and methods. The study was a retrospective single-center observational analysis of the patients with advanced melanoma, treated with electrochemotherapy after previous IFN-a adjuvant therapy. Five patients, treated between January 2008 and December 2014, were included into the study, regardless of the time point of IFN-a adjuvant therapy.

Results. Electrochemotherapy of recurrent melanoma after the IFN-a adjuvant therapy proved to be a safe and effective treatment. Patients with one or two metastases responded completely. Among patients with multiple metastases, there was a variable response rate. In one patient all 23 metastases responded completely, in second patient more than $85 \%$ of all together 80 metastases responded completely and in third patient all 5 metastases had partial response. Taking into account all metastases from all patients together there was an $85 \%$ complete response rate.

Conclusions. The study showed that electrochemotherapy of recurrent melanoma after the IFN-a adjuvant therapy is a safe and effective treatment modality, which results in a high complete response rate, not only in single metastasis, but also in multiple metastases. The high complete response rate might be due to an IFN-a immune-editing effect, however, further studies with a larger number of patients are needed to support this presumption.

Key words: electrochemotherapy; melanoma; IFN-a

\section{Introduction}

Melanoma is, due to the high risk of metastases development and its resistance to different treatment strategies, still the most lethal skin cancer. The most effective treatment for primary melanoma of stage I and II is its removal by radical surgical excision with the associated safety margin, usually followed by a sentinel lymph node biopsy. Although this approach is curative in many cases, relapse with disseminated disease occurs in some patients. Therefore, in patients with an increased risk for re- current disease adjuvant immunotherapy might be applied.1,2

The sole recognized postsurgical adjuvant therapy still is interferon alpha (IFN- $\alpha$ ). In several clinical studies both interferon types, the highdose interferon alpha- $2 b$ (IFN- $\alpha 2 b$ ) and low-dose interferon alpha-2a (IFN- $\alpha 2 \mathrm{a})$ were shown to have significant effect on progression-free survival. ${ }^{3-5}$ Recent meta-analysis showed statistically significant improvement in disease free survival and overall survival in patients with high risk melanoma (stage Ilb-IIIC according to The American 
Joint Committee on Cancer [AJCC] TNM Cancer Staging Manual $7^{\text {th }}$ edition ${ }^{6}$ ) treated with adjuvant IFN- $\alpha$ after surgery. ${ }^{7,8}$

When melanoma recurs, other treatment modalities are needed for local or systemic control of the disease. Most commonly used are systemic chemotherapy with dacarbazine, irradiation, isolated limb perfusion or electrochemotherapy and in recent years also new targeted therapies with BRAF and/or MEK inhibitors and antibodies against CTLA-4. ${ }^{9,10}$

Electrochemotherapy is one of the treatment modalities for local treatment of malignant melanoma, which is using electroporation as a delivery system for the chemotherapeutic drugs bleomycin or cisplatin into the tumor. ${ }^{11-14}$ Under the high external electric field the plasma membrane becomes permeable, thus facilitating drug delivery into the tumors. Numerous clinical studies have demonstrated the effectiveness of electrochemotherapy on a variety of cutaneous and also deep seated tumors, such as liver metastases of colorectal cancer..$^{15}$ Among cutaneous tumors, electrochemotherapy is very effective in treatment of melanoma, with a complete response rate after a single treatment of $74 \%{ }^{16}$ Some studies have recently reported on beneficial effect on melanoma treatment after combining electrochemotherapy with new targeted therapies such as dabrafenib or ipilimumab. . $^{1718}$ Furthermore, electrogene therapy with plasmids coding for interleukin-12 (IL-12) or antiangiogenic molecules, is also in clinical testing for melanoma treatment. ${ }^{19-22}$

In preclinical studies, it was shown that adjuvant therapy with TNF- $\alpha$, IL-2, IL-12 and CpG oligonucleotides might boost electrochemotherapy response. ${ }^{23-30}$ However, the role of adjuvant IFN- $\alpha$ has not been explored yet, neither on preclinical or clinical level. Therefore, the aim of the study was to evaluate the safety and effectiveness of electrochemotherapy on recurrent melanoma after IFN- $\alpha$ adjuvant therapy of melanoma patients.

\section{Patients and methods}

\section{Study design and patient selection}

The study was conducted as a retrospective singlecenter analysis of patients with advanced malignant melanoma treated with electrochemotherapy, who previously received IFN- $\alpha$ adjuvant therapy, after surgery of primary melanoma. All the patient files where electrochemotherapy was performed in the last 6 years, between January 2008 and
December 2014, were reviewed regardless of the time point of IFN- $\alpha$ adjuvant therapy. Among all the (50) patients treated with electrochemotherapy in that time period only 5 of them met the requirements and were further investigated. The trial was approved by an Institutional Review Board and National Medical Ethic Committee 97/06/02. The patients signed the informed consent before the treatment.

\section{Investigated entities}

First the patient's general characteristics (gender, age), the site and TNM, pathological stage (AJCC TNM Cancer Staging Manual, 7th edition) and Breslow thickness of primary melanoma were recorded. Than therapeutic dose and duration of IFN- $\alpha$ adjuvant therapy were recorded for each patient. The treatment free-interval between the end of IFN- $\alpha$ adjuvant therapy and electrochemotherapy treatment was calculated. The site and number of melanoma nodules treated with electrochemotherapy were further recorded and afterwards effectiveness of electrochemotherapy was evaluated.

\section{IFN- $\alpha$ adjuvant therapy}

All five patients received IFN- $\alpha$ as a post-surgical adjuvant therapy. Patient 1 and 2 received lowdose IFN- $\alpha$ since the IFN- $\alpha$ adjuvant therapy was administered before 2010, when new guidelines for melanoma treatment at the Institute of Oncology Ljubljana were accepted. Patient 1 and 2 received Roferon-A® (interferon Alfa-2a) (Roche, Basel, Switzerland) subcutaneously at a dose of 3 or 6 million IU three times a week (Table 2) for 33 and 7 months, respectively, according to the instructions of an oncologist. Patient 3, 4 and 5 received high dose interferon Intron ${ }^{\circledR}$ A (interferon a- alpha $2 b$ ) (Merck, Kenilworth, New Jersey, USA) according to the Kirkwood scheme. ${ }^{31}$ The exact schedule and the dose was adjusted for each patient by his oncologist (Table 2).

\section{Electrochemotherapy}

Patients were treated according to the Standard Operating Procedure (SOP) for electrochemotherapy. ${ }^{16}$ Briefly, electrochemotherapy of cutaneous melanoma nodules was performed using either intravenous bleomycin (Bleomedac, Medac, Wedel, Germany) in a dose of $15,000 \mathrm{IU} / \mathrm{m}^{2}$ or intratumoral cisplatin (Cisplatin, Medac) injection in a concentration of $2 \mathrm{mg} / \mathrm{ml}$ and dose is applied according 
TABLE 1. Patients' characteristics

\begin{tabular}{|c|c|c|c|c|c|}
\hline Gender & Male & Male & Female & Female & Female \\
\hline TNM* & T3aNOMO & T2aNOMO & T4bNlaMO & T3aNlaMo & T4bNlaMO \\
\hline Patological stage* & Stage lla & Stage $\mathrm{lb}$ & Stage IIIb & Stage Illa & Stage IIIb \\
\hline Localisation of primary tumor & Right lower leg & Left foot & Right foot & Right lower leg & Back \\
\hline Localisation of metastases treated with ECT & Right lower leg & Left foot (dorsum) & Left lower leg & Right lower leg & Breast, left side \\
\hline
\end{tabular}

* according to AJCC TNM Cancer Staging Manual $7^{\text {th }}$ edition $(2010)^{6}$; ECT $=$ electrochemotherapy

to ESOPE protocol. ${ }^{16}$ Standard pulse parameters for electrochemotherapy (voltage to distance ratio $1300 \mathrm{~V} / \mathrm{cm}, 8$ pulses, $100 \mu \mathrm{s}, 5000 \mathrm{~Hz}$ ) were used. ${ }^{16}$ Electric pulses were generated by Cliniporator pulse generator (IGEA, s.r.l., Carpi, Italy) and delivered by parallel stainless steel plate electrodes with 6 or $8 \mathrm{~mm}$ distance in between. Electric pulses were applied to the tumors nodules in a way so as to cover the whole tumor area, including the safety margin.

\section{Response assessment}

Antitumor efficacy was evaluated 4 weeks after electrochemotherapy, patient were then monitored monthly. Treatment response was defined either as complete response (CR), when the tumor was not palpable, partial response (PR), when the tumor decreased more than $50 \%$ of the measurable lesions; no change (NC), when tumor decreased less than $50 \%$ or increased up to $25 \%$, or progressive disease (PD), when tumor increased for more than $25 \%$. Determination was based on criteria of WHO Handbook for Reporting Results of Cancer Treatment where for all response definitions minimum 4-week duration was required for qualifying the response.

\section{Results}

\section{Patients' characteristics}

Only 5 patients fulfilled all the requirements for inclusion into this retrospective study. Among them there were two male and three female patients with a median age of electrochemotherapy treatment 71 years (range from 50-76 years). TNM and patho- logical stage were recorded for all five patients, as well as Breslow thickness (Table 1). The localizations of primary tumors were on the upper leg, foot or the back (Table 1). All the patients were identified as patients with high risk of recurrence and were therefore assigned for IFN- $\alpha$ adjuvant therapy. The relapse of melanoma occurred in all five patients. Recurrence time was variable among patients; from a few months to a few years (Table 1). Other comorbidities were also recorded for all 5 patient; only patient 2 had arterial hypertension and no other comorbidities were recorded.

\section{IFN- $\mathrm{a}$ adjuvant therapy and disease progression}

Adjuvant therapy with IFN- $\alpha$ in our investigated patients can be divided into two subtypes; lowdose treatment for patients 1 and 2 and high dose treatment for another three patients (Table 2). After the completed adjuvant therapy with IFN- $\alpha$, in all five patients disease had progressed to a metastatic disease. Disease free interval, progression of the disease and treatment procedures vary for each patient (Table 2). Based on the decision of an institutional committee for melanoma treatment, electrochemotherapy was offered to the patients as another treatment option after several surgical excisions and in patient 1 and 2 also irradiation.

- In patient 1 inguinal lymph node metastasis occurred after a disease free interval and inguinal and retroperitoneal lymph node dissection was performed thereafter. The patient was irradiated as well. When progression of the disease occurred 3 years later, two metastases were excised within 2 months and the patient was irradiated again. In 2 months new metastases, 
TABLE 2. Treatment regime

\begin{tabular}{|c|c|c|c|c|c|}
\hline INF-a dose & $\begin{array}{l}6 \text { million IU } 3 x \\
\text { weekly (s.c.) }\end{array}$ & $\begin{array}{l}3 \text { million IU } 3 \text { x weekly } \\
\text { (s.c.) }\end{array}$ & $\begin{array}{l}35 \text { milion IU } 20 x \\
\text { (i.v.) in } 8 \text { weeks/ } \\
15 \text { million IU } 3 x \\
\text { weekly (s.c.) for } 13 \\
\text { weeks/ } 10 \text { milion IU } \\
3 \times \text { weekly (s.c.) for } \\
13 \text { weeks* }\end{array}$ & $\begin{array}{l}30 \text { milion IU } 20 \times \text { (i.v.) } \\
\text { in } 4 \text { weeks followed } \\
\text { by } 15 \text { million IU } 3 \times \\
\text { weekly (s.c.) for } 35 \\
\text { weeks followd by } 10 \\
\text { milion IU } 3 \times \text { weekly } \\
\text { (s.c.) for } 12 \text { weeks }\end{array}$ & $\begin{array}{l}30 \text { milion IU } 9 \times \text { (i.v.) } \\
\text { in } 2 \text { weeks followed } \\
\text { by } 20 \text { milion IU } 11 x \\
\text { (i.v.) in } 3 \text { weeks } \\
\text { followed by } 15 \text { million } \\
\text { IU } 3 x \text { weekly (s.c.) for } \\
13 \text { weeks** }\end{array}$ \\
\hline Disease free interval & 4 months & 5 years 6 months & 6 months & 3 months & 2 months \\
\hline $\begin{array}{l}\text { Surgically excised } \\
\text { metastases+ }\end{array}$ & Yes & Yes & No & Yes & Yes \\
\hline $\begin{array}{l}\text { Interval between INF-a and } \\
\text { ECT treatment }\end{array}$ & 4 years 8 months & 12 years 11 months & 7 months & 6 months & 7 months \\
\hline Number of lesions & 2 & 1 & 80 & 23 & 5 \\
\hline Size of the nodules & $\begin{array}{l}1.0 \times 1.5 \mathrm{~cm} \\
1.5 \times 1.5 \mathrm{~cm}\end{array}$ & $3 \times 3 \mathrm{~cm}$ & $0.3-1 \mathrm{~cm}$ & $0.1-0.8 \mathrm{~cm}$ & $0.7-1.5 \mathrm{~cm}$ \\
\hline Effect after 4 weeks & $\mathrm{CR}$ & $\mathrm{CR}$ & $>85 \% \mathrm{CR}$ & All CR & $100 \%$ PR \\
\hline LRD*** or date of death (D) & April 2010 (LRD) & December 2014 (LRD) & April 2014 (LRD) & December 2013 (D) & March 2013 (D) \\
\hline
\end{tabular}

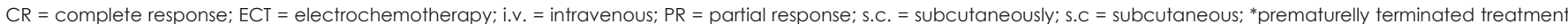
due to ineffective treatment; ** intravenous dose (i.v. dose) was decreased to 20 million IU due to pathological liver tests - prematurelly terminated treatment due to side effects,

*** after last record date at the Institute of Oncology Ljubljana patients were given only paliative care at their regional centers, ${ }^{+}$details on localization and number of excised metastates in paragraph Pacients' caracteristics.

which were treated with electrochemotherapy, occurred.

- In patient 2 after a disease free interval a metastasis on the limb occurred and was immediately excised. Four years later, another excision with following inguinal dissection and irradiation was performed. In the following 2 years three in-transit metastases were excised from the dorsum of the left foot. A new metastasis occurred 1 year after the last in-transit metastasis excision, which was then treated with electrochemotherapy.

- Disease free interval for the patient 3 was 6 months; thereafter multiple metastases occurred on the limb and were treated with electrochemotherapy.

- In patient 4 a metastasis occurred on the scar of a primary excised melanoma only 3 months after completing adjuvant therapy with IFN- $\alpha$. It was immediately excised, although PET/CT scan and thin needle biopsy showed multiple metastases in the same area, which were then treated with electrochemotherapy.

- In patient 5 the disease-free interval was 2 months, followed by two excisions of metasta- ses and shortly afterwards electrochemotherapy of 5 metastases on the trunk. Due to partial response of all 5 metastases, these metastases and newly formed metastases on the trunk were again treated with electrochemotherapy 6 weeks after the first treatment.

\section{Electrochemotherapy following IFN- $\mathrm{a}$ adjuvant therapy}

At the time of electrochemotherapy patient 2 was presented with a single metastasis on the limb, whereas patients 1, 3, 4 and 5 were presented with multiple metastases on the limb (patient 1, 3 and 4 ) or trunk (patient 5). All metastases present at the time of electrochemotherapy were treated. Electrochemotherapy was effective in all five patients, with a variable response rate (Table 2 ).

In patient 1 and 2 cisplatin was given intratumoraly due to previous irradiation of the patients. In some studies, it was reported that previous irradiation can cause lower effectiveness of i.v. electrochemotherapy. ${ }^{32}$ Fibrosis can be one of the causes for lower effectiveness. Less fluid in the tissue results in less lymphatic infiltration and also lower 
current in the nodule and can therefore contribute to the lower effectiveness of electrochemotherapy of pre-iradiated tissues. Intratumoral injection of chemotherapeutic drug can overcome those obstacles and can results in higher effectiveness.

Electrochemotherapy following IFN- $\alpha$ adjuvant therapy was effective treatment modality, regardless of drug used for electrochemotherapy, bleomycin or cisplatin. Single metastasis responded completely, while multiple metastases had a variable response rate. In patient 4 all 23 metastases responded completely, in patient 3 more than $85 \%$ of all together 80 metastases responded completely and in patient 5 all 5 metastases had partial response. Taking into account all metastases from all patients together there was an $85 \%$ complete response rate. After electrochemotherapy no side effects, such as local erythema, bleeding, infection on the site of electrochemotherapy, or muscle contractions, were reported. Nevertheless new metastases mostly occurred within 1 month (patients 1, 3 and 5) or 2 months (patient 4) after the treatment. In patient 2 , with a single metastasis at the time of electrochemotherapy, new metastases occurred after 1 year and 10 months. In patient 1 additional electrochemotherapy of 14 new lesions was performed thereafter, which also resulted in $100 \%$ complete response. Nevertheless the disease progressed and although systemic chemotherapy with dacarbazine was administered, new distant metastases in the head and neck region occurred. Electrochemotherapy was then used for palliative care. In patient 2 disease also progressed and due to several metastases, isolated limb perfusion was performed 4 years after electrochemotherapy. No new metastasis occurred yet. Dacarbazine and later also ipilimumab were prescribed for patient 3 with progressive metastatic disease, with metastases present also in liver and lungs. In patient 4 new subcutaneous metastases were effectively irradiated and year after brain metastasis occurred. Electrochemotherapy response in all those patients (patient 1-4) remains the same during the whole observational period. Due to partial response of all 5 metastases in patient 5 , these metastases and 12 newly formed metastases on the trunk were again treated with electrochemotherapy, 6 weeks after the first treatment. After the second electrochemotherapy treatment all 17 metastases (including 5 retreated metastases) responded completely. The patient was later treated with dacarbazine and vemurafenib due to soft tissue and lung metastases, but disease progressed with new metastases in lungs and brain.
Before ECT
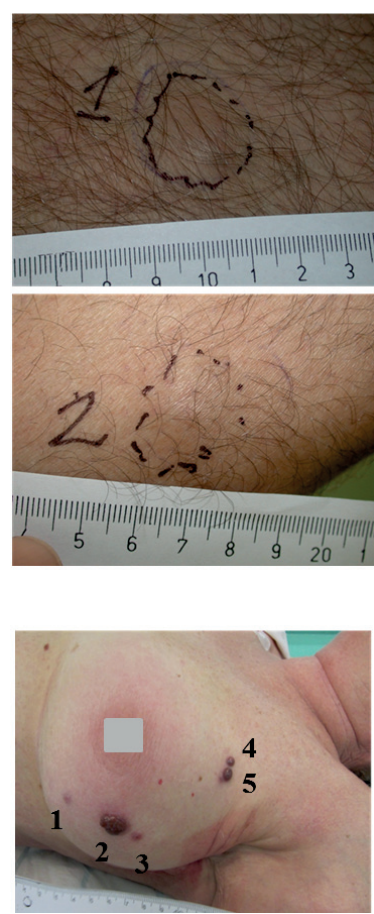

FIGURE 1. Melanoma lesions in patient 1 (A) and patient 5 (B) before and 6 weeks after electrochemotherapy (ECT). Both metastases in patient 1 responded completely, while in patient 5 although the crust is seen on the images there was nodule under it and response was evaluated as partial response.

\section{Discussion}

This is the first study to our knowledge which discusses the effectiveness of electrochemotherapy after IFN- $\alpha$ adjuvant therapy for treatment of melanoma metastases.

In recent years electrochemotherapy has been widely used in clinical studies for treatment of cutaneous and also deep seated tumors. Among skin cancers electrochemotherapy was very effective in the treatment of malignant melanoma, with a complete response rate after single treatment $74 \%$, according to ESOPE study. ${ }^{16}$ Although the electrochemotherapy response rate is quite high, and effective on most of tumor histologies, recently there is some evidence that there is a variability in the response rates of different tumor histologies. The meta-analysis by Mali et al. has shown that melanoma tumors are less responsive than non-melanoma tumors. The effectiveness of electrochemotherapy was tumor type dependent, namely the complete response rate was $57 \%$ of melanoma tumors and $67 \%$ of other, non-melanoma tumors. Among carcinomas, basal cell carcinoma was the most responsive tumor type, with up to $89 \%$ complete responses. ${ }^{33}$ 
Furthermore, the importance of the immune response elicited by electrochemotherapy locally was explored. ${ }^{34,35}$ Immunogenic cell death of cancer cells was proposed to contribute to the curability of the treated metastases. The concept of immunogenic cell death, which is triggered by some cancer therapies, is initiated by damage-associated molecular patterns, which can further trigger an adaptive immune response against tumors. ${ }^{34}$ Some pre-clinical studies have explored the possibility of adjuvant electrogene therapy with plasmid encoding IL-12, which greatly increase the response rate of the electrochemotherapy treated tumors. ${ }^{30}$ The recent clinical study, investigating the combined treatment of ipilimumab and electrochemotherapy has shown a better response than ipilimumab alone. ${ }^{17}$

In this report we show that electrochemotherapy was safe and effective also after IFN- $\alpha$ therapy. IFN- $\alpha$, although given to the patients in different periods before electrochemotherapy may also contribute to the response rate of the electrochemotherapy treated melanoma metastases. Namely, response rate in patients with electrochemotherapy after adjuvant IFN- $\alpha$ was $100 \%$ partial response (patient 5) or from $85 \%$ to $100 \%$ complete response (patient 1, 3 and 4) in patients with multiple metastases, which is an equal or even higher percentage than demonstrated in previous studies; $85 \%$ of metastases responded completely in the present study, while the results of meta-analysis showed that $57 \%$ of melanoma metastases responded completely. ${ }^{16,33}$

We might speculate that the effectiveness may be increased by the previous immunostimulatory IFN- $\alpha$ adjuvant therapy, which would be reflected in high response rate of the treated tumors. IFN- $\alpha$ is one of the type I interferons, an important interferon family, involved in immune-editing process. Their main importance is the effect on the hematopoietic cells; induction of bystander $\mathrm{T}$ cell proliferation, long-term survival and expression of antiapoptotic genes. ${ }^{36-37}$ Furthermore, interferons have also great impact on maturation and differentiation of dendritic cells, cells which are considered to be the most effective antigen presenting cells. ${ }^{34}$ Taking all this findings into account, IFN- $\alpha$ might have a significant role in a link between the innate and adaptive immune system. Similarly, new targeted therapy with ipilimumab acts on dendritic cells - cytotoxic T lymphocytes (CTLs) interaction. Dendritic cells are presenting tumor antigens to CTLs, which can then destroy cancer cells. But along with tumor antigens the dendritic cells present also an inhibitory signal, which can bind to a receptor on the CTLs; cytotoxic T lymphocyte-associated antigen 4 (CTLA-4) and thereby block the cytotoxicity of CTL. Ipilimumab binds to CTLA-4 and block the inhibitory signal. ${ }^{38,39}$

One of the possible reasons for the very good efficiacy of electrochemotherapy, following IFN- $\alpha$ adjuvant therapy is immune system activation by electrochemotherapy, which was previously modulated by IFN- $\alpha .{ }^{40}$ Calvet et al. demonstrated in an in vivo preclinical study that electrochemotherapy not only has a cytotoxic effect towards cancer cells, but can also generate a systemic anticancer immune response, with imunogenic cell death. 33,40 Dying cancer cells then behave as a therapeutic vaccine, leading to a cytotoxic immune response against remaining tumor cells. ${ }^{35,42}$ It was also demonstrated that electrochemotherapy is more effective in immunocompetent mice, causing complete tumor regression, whereas in immunodeficient mice the complete response was not obtained. ${ }^{35}$

The drawback of our study is that we have no data on the immune status of the patients and that this is an observational study. Nevertheless, although this group of patients is small it might indicate on the potential of combining immunostimulatory treatments with electrochemotherapy, which can be explored in different ways. One of the recent ideas is that electrochemotherapy can serve as a vaccination to adjuvant peritumoral immunostimulatory therapy that can boost the local effect as well it may have the systemic effect. ${ }^{42}$

\section{Conclusions}

The report demonstrates that combining electrochemotherapy with preceded IFN- $\alpha$ adjuvant therapy is a safe and effective treatment modality, which results in high complete response rate, not only in single metastasis, but also in multiple metastases. The high complete response rate might be due to IFN- $\alpha$ immune-editing effect, however further controlled studies on a larger number of patients are needed to support this presumption.

\section{Acknowledgements}

We greatly appreciate the help of our research nurse Tjasa Pecnik, B. Sc. The research was financially supported from the Slovenian Research Agency (program no. P3-0003). This manuscript is a result of the networking efforts of the COST Action TD1104 (www.electroporation.net). Research 
was conducted in the scope of EBAM European Associated Laboratory (LEA).

\section{References}

1. Veronesi U, Adamus J, Aubert C, Bajetta E, Beretta G, Bonadonna G, et al. A randomized trial of adjuvant chemotherapy and immunotherapy in cutaneous melanoma. N Engl J Med 1982; 307: 913-6.

2. Pflugfelder A, Kochs C, Blum A, Capellaro M, Czeschik C, Dettenborn T, et al. Malignant melanoma S3-guideline "diagnosis, therapy and follow-up of melanoma". J Dtsch Dermatol Ges 2013; 11 (Suppl 6): 1-116, 1-126.

3. Hauschild A. Adjuvant interferon alfa for melanoma: new evidence-based treatment recommendations? Curr Oncol 2009; 16: 3-6.

4. Cole BF, Gelber RD, Kirkwood JM, Goldhirsch A, Barylak E, Borden E. Qualityof-life-adjusted survival analysis of interferon alfa-2b adjuvant treatment of high-risk resected cutaneous melanoma: an Eastern Cooperative Oncology Group study. J Clin Oncol 1996; 14: 2666-73.

5. Grob JJ, Dreno B, de la Salmonière $P$, Delaunay $M$, Cupissol $D$, Guillot $B$, et al. Randomised trial of interferon alpha-2a as adjuvant therapy in resected primary melanoma thicker than $1.5 \mathrm{~mm}$ without clinically detectable node metastases. French Cooperative Group on Melanoma. Lancet 1998; 351: 1905-10.

6. Edge SB, Compton CC. The American Joint Committee on Cancer: the 7th edition of the AJCC cancer staging manual and the future of TNM. Ann Surg Oncol 2010; 17: 1471-4.

7. Mocellin S, Pasquali S, Rossi CR, Nitti D. Interferon alpha adjuvant therapy in patients with high-risk melanoma: a systematic review and meta-analysis. J Natl Cancer Inst 2010; 102: 493-501.

8. Mocellin S, Lens MB, Pasquali S, Pilati P, Chiarion Sileni V. Interferon alpha for the adjuvant treatment of cutaneous melanoma. Cochrane Database Syst Rev 2013; 6: CD008955.

9. Kaufman HL, Kirkwood JM, Hodi FS, Agarwala S, Amatruda T, Bines SD, et al. The Society for Immunotherapy of Cancer consensus statement on tumour immunotherapy for the treatment of cutaneous melanoma. Nat Rev Clin Oncol 2013; 10: 588-98.

10. Karimkhani C, Gonzalez R, Dellavalle RP. A review of novel therapies for melanoma. Am J Clin Dermatol 2014; 15: 323-37.

11. Sersa G, Miklavcic D, Cemazar M, Rudolf Z, Pucihar G, Snoj M. Electrochemotherapy in treatment of tumours. EJSO 2008; 34: 232-40.

12. Testori A, Rossi CR, Tosti G. Utility of electrochemotherapy in melanoma treatment. Curr Opin Oncol 2012; 24: 155-61.

13. Cadossi R, Ronchetti M, Cadossi M. Locally enhanced chemotherapy by electroporation: clinical experiences and perspective of use of electrochemotherapy. Future Oncol 2014; 10: 877-90.

14. Testori A, Faries MB, Thompson JF, Pennacchioli E, Deroose JP, van Geel AN, et al. Local and intralesional therapy of in-transit melanoma metastases. $J$ Surg Oncol 2011; 104: 391-6.

15. Edhemovic I, Brecelj E, Gasljevic G, Marolt Music M, Gorjup V, Mali B, et al. Intraoperative electrochemotherapy of colorectal liver metastases. I Surg Oncol 2014; 110: 320-7.

16. Marty M, Sersa G, Garbay J, Gehl J, Collins C, Snoj M, et al Electrochemotherapy - An easy, highly effective and safe treatment of cutaneous and subcutaneous metastases: Results of ESOPE (European Standard Operating Procedures of Electrochemotherapy) study. Eur J Cancer Suppl 2006; 4: 3-13.

17. Simeone E, Benedetto L, Gentilcore G, Capone M, Caraco C, Di Monta G, et al. Combination therapy with ipilimumab and electrochemotherapy; preliminary efficacy results and correlation with imunological parameters. $J$ Transl Med 2014; 12 (Suppl 1): 05

18. Valpone S, Campana L, Pigozzo J, Chiarion-Sileni V. Consolidation electrochemotherapy with bleomycin in metastatic melanoma during treatment with dabrafenib. Radiol Oncol 2015; 49: 71-4.

19. Cemazar M, Jarm T, Sersa G. Cancer electrogene therapy with interleukin-12. Curr Gene Ther 2010; 10: 300-11.

20. Daud Al, DeConti RC, Andrews S, Urbas P, Riker Al, Sondak VK, et al. Phase I trial of interleukin-12 plasmid electroporation in patients with metastatic melanoma. J Clin Oncol 2008; 26: 5896-903.
21. Spanggaard I, Snoj M, Cavalcanti A, Bouquet C, Sersa G, Robert C, et al. Gene electrotransfer of plasmid antiangiogenic metargidin peptide (AMEP) in disseminated melanoma: safety and efficacy results of a phase I first-in-man study. Hum Gene Ther Clin Dev 2013; 24: 99-107.

22. Heller LC, Heller R. Electroporation gene therapy preclinical and clinical trials for melanoma. Curr Gene Ther 2010; 10: 312-7.

23. Cemazar M, Todorovic V, Scancar J, Lampreht U, Stimac M, Kamensek U, et al. Adjuvant TNF- $\alpha$ therapy to electrochemotherapy with intravenous cisplatin in murine sarcoma exerts synergistic antitumor effectiveness. Radio Oncol 2015; 49: 32-40.

24. Gerlini G, Di Gennaro P, Borgognoni L. Enhancing anti-melanoma immunity by electrochemotherapy and in vivo dendritic-cell activation. Oncoimmunology 2012; 1: 1655-7.

25. Sersa G, Cemazar M, Menart V, Gaberc-Porekar V, Miklavcic D. Anti-tumor effectiveness of electrochemotherapy with bleomycin is increased by TNFalpha on SA-1 tumors in mice. Cancer Lett 1997; 116: 85-92.

26. Heller L, Pottinger C, Jaroszeski MJ, Gilbert R, Heller R. In vivo electroporation of plasmids encoding GM-CSF or interleukin-2 into existing B16 melanomas combined with electrochemotherapy induces long-term antitumour immunity. Melanoma Res 2000; 10: 577-83.

27. Mir LM, Roth C, Orlowski S, Belehradek J, Fradelizi D, Paoletti $C$, et al. Potentiation of the antitumoral effect of electrochemotherapy by immunotherapy with allogeneic cells producing interleukin 2. C R Acad Sci III 1992; 314: 539-44.

28. Mir LM, Orlowski S, Poddevin B, Belehradek J. Electrochemotherapy tumor treatment is improved by interleukin-2 stimulation of the host's defenses. Eur Cytokine Netw 1992; 3: 331-4.

29. Roux S, Bernat C, Al-Sakere B, Ghiringhelli F, Opolon P, Carpentier AF, et al. Tumor destruction using electrochemotherapy followed by CpG oligodeoxynucleotide injection induces distant tumor responses. Cancer Immunol Immunother 2008; 57: 1291-300.

30. Sedlar A, Dolinsek T, Markelc B, Prosen L, Kranjc S, Bosnjak M, et al. Potentiation of electrochemotherapy by intramuscular IL-12 gene electrotransfer in murine sarcoma and carcinoma with different immunogenicity. Radiol Oncol 2012; 46: 302-11.

31. Moreno Nogueira JA, 1 Valero Arbizu M, Pérez Temprano R. Adjuvant treatment of melanoma. ISRN Dermatol. 2013; 2013: 545631.

32. Groselj A, Kos B, Cemazar M, Urbancic J, Kragelj G, Bosnjak M, Veberic B, Strojan $P$, Miklavcic D, Sersa G. Coupling treatment planning with navigation system: a new technological approach in treatment of head and neck tumors by electrochemotherapy. Biomed Eng Online. 2015; 14 Suppl 3: S2.

33. Mali B, Jarm T, Snoj M, Sersa G, Miklavcic D. Antitumor effectiveness of electrochemotherapy: a systematic review and meta-analysis. EJSO 2013; 39: 4-16.

34. Calvet CY, Famin D, André FM, Mir LM. Electrochemotherapy with bleomycin induces hallmarks of immunogenic cell death in murine colon cancer cells. Oncoimmunology 2014; 3: e28131.

35. Sersa G, Miklavcic D, Cemazar M, Belehradek J, Jarm T, LM. Mir Electrochemotherapy with CDDP on LPB sarcoma: comparison of the anti-tumor effectiveness in immunocompetent and immunodeficient mice. Bioelectrochem Bioenerg 1997; 43: 279-83.

36. Tough DF, Borrow P, Sprent J. Induction of bystander T cell proliferation by viruses and type I interferon in vivo. Science 1996; 272: 1947-50.

37. Paquette RL, Hsu NC, Kiertscher SM, Park AN, Tran L, Roth MD, et al. Interferon-alpha and granulocyte-macrophage colony-stimulating factor differentiate peripheral blood monocytes into potent antigen-presenting cells. J Leukoc Biol 1998; 64: 358-67.

38. Melero I, Hervas-Stubbs S, Glennie M, Pardoll DM, Chen L. Immunostimulatory monoclonal antibodies for cancer therapy. Nat Rev Cancer 2007; 7: 95-106.

39. Hodi FS, O'Day SJ, McDermott DF, Weber RW, Sosman JA, Haanen JB, et al. Improved survival with ipilimumab in patients with metastatic melanoma. N Engl J Med 2010; 363: 711-23.

40. O'Brien MA, Power DG, Clover AJ, Bird B, Soden DM, Forde PF. Local tumour ablative therapies: opportunities for maximising immune engagement and activation. Biochim Biophys Acta 2014; 1846: 510-23.

41. Sersa G, Kotnik V, Cemazar M, Miklavcic D, Kotnik A. Electrochemotherapy with bleomycin in SA-1 tumor-bearing mice--natural resistance and immune responsiveness. Anticancer Drugs 1996; 7: 785-91.

42. Sersa G, Teissie J, Signori E, Kamensek U, Marshall G, Cemazar M, et al Electrochemotherapy of tumors as in situ vaccination boosted by immunogene electrotransfer. Cancer Immunol Immunother 2015; 64: 1315-27. 